\title{
Cooling the cervix below the body temperature may reduce the risk of preterm birth
}

\begin{abstract}
Premature birth causes high rates of neonatal mortality and morbidity. Preterm birth is a global problem; with a prevalence of 8 to $12 \%$. My hypothesis; "cooling the cervix below the body temperature may help to reduce preterm birth by its anti-inflammatory effect". There is no study in the literature so far that suggests this hypothesis. Out of all of the suspected causes of preterm labour, inflammation and/or infection are the only pathological process for which both a link with preterm birth has been established and a molecular pathophysiology defined. Inflammation has been implicated in the mechanisms responsible for preterm and term parturition, as well as fetal injury. Mild hypothermia decreases inflammatory responses in both brain inflammation and stroke, implicating a direct anti-inflammatory effect of cooling. It is essential that before embarking on a human study, animal studies should be done to support this hypothesis. If my hypothesis is true this may improve neonatal outcome by reducing neonatal death and major morbidity.
\end{abstract}

Volume 2 Issue 4 - 2015

\author{
Berrin Tezcan \\ Department of Obstetrics and Gynaecology, Newham \\ University Hospital, UK
}

\begin{abstract}
Correspondence: Berrin Tezcan, Department of Obstetrics and Gynaecology, Newham University Hospital, 10 Dulwich Common, SE2I 7EX, UK, Tel 44 7962645843,

Email tezcanberrin@hotmail.com
\end{abstract}

Received: February 13, 2015 | Published: July 13, 2015

\section{Introduction}

Premature birth causes high rates of neonatal mortality and morbidity. Preterm birth is a global problem; with a prevalence of 8 to $12 \%$. There are multiple causes of preterm birth. Inflammation has been implicated in the mechanisms responsible for preterm and term delivery. Out of all of the suspected causes of preterm labour, inflammation and/or infection is the only pathological process for which both a link with preterm birth has been established and a molecular pathophysiology defined..$^{1,2}$ Inflammation has also been implicated in the mechanism of spontaneous delivery at term. Most cases of histological chorioamnionitis and histopathological inflammation, both in preterm and term labour, are sub-clinical in nature. One recent study has shown that women with PPROM before 27 weeks have a 9\% recurrence risk of early PPROM and a risk of $35 \%$ of having a preterm delivery in a subsequent pregnancy. ${ }^{3}$ Mild hypothermia decreases inflammatory responses in both brain inflammation and stroke, implicating a direct anti-inflammatory effect of cooling.

\section{The hypothesis}

This is a theoretical paper. There is no study in the literature so far that suggests this hypothesis. This hypothesis has never been reported to date. In order to support this hypothesis, before embarking on a human study, animal studies should be consideredas proof of principle study.

\section{Aim}

To design a device to cool the cervix below the body temperature $\left(33-34^{\circ} \mathrm{C}\right)$ and to reduce the risk of preterm birth. The group of patients who are high risk for preterm birth, symptomatic for preterm birth and/or fFN positive will be included and will be grouped into two. The device will be applied/placed on group1 to cool the cervix and group2 will be the control group. I don't have an established idea yet on achieving mild hypothermia on the cervix but I believe we could design a device that will circulate liquid ice to achieve the targeted temperature around $33-34^{\circ} \mathrm{C}$ and vagina will be able to hold the device in place. Mild hypothermia decreases inflammatory responses in both brain inflammation and stroke, implicating a direct anti-inflammatory effect of cooling.

\section{Discussion}

Cervical cerclage is commonly used in the management of women considered to be at high risk of spontaneous preterm birth and second-trimester loss. Insertion is dictated by factors such as uterine anomalies, multiple pregnancies, a history of cervical trauma and cervical shortening diagnosed on transvaginal ultrasound examination. However, its efficacy and use in these different groups is very controversial because there is contradiction in the results of individual studies and meta-analyses. ${ }^{4}$ Several large trials and systematic reviews have shown progestogens to be effective in preventing or delaying preterm birth in selected high risk women with a singleton pregnancy, including those with previous preterm birth or a short cervix. Although an improvement in short term neonatal outcomes has been shown in some trials these have not consistently been confirmed in meta-analyses. Additionally data on longer term outcomes is limited to a single trial where no difference in outcomes was demonstrated at four years of age of the child, despite those in the "progesterone" group having a lower incidence of preterm birth. ${ }^{5}$

Enzymes are very efficient catalysts for biochemical reactions. They speed up reactions by providing an alternative reaction pathway of lower activation energy. As the temperature rises, reacting molecules have more and more kinetic energy. This increases the chances of a successful collision and so the rate increases. There is a certain temperature at which an enzymes catalytic activity is at its greatest. This optimal temperature is usually around human body temperature $\left(37.5^{\circ} \mathrm{C}\right)$ for the enzymes in human cells. Above this temperature the enzyme structure begins to break down since at higher temperatures intra and intermolecular bonds are broken as the enzyme molecules gain even more kinetic energy.

Every enzyme has a temperature range of optimum activity. Outside the temperature range the enzyme is rendered inactive and is said to be totally inhibited. This occurs because as the temperature changes this supplies enough energy to break some of the intra molecular attraction between polar groups (hydrogen bonding, dipole-dipole attractions) 
as well as the hydrophobic forces between non-polar groups within the protein structure. When these forces are disturbed and changed, this causes a change in the secondary and tertiary levels of protein structure, and the active site is altered in its catalyze. Most enzymes (and there are hundreds within the human organism) within the human cells will shut down at a body temperature below a certain value which varies according to each individual. This can happen if body temperature gets too low (hypothermia) or too high (hyperthermia).

Mild hypothermia decreases inflammatory responses in both brain inflammation and stroke, implicating a direct anti-inflammatory effect of cooling. Therapeutic hypothermia (TH) is nowadays one of the most important methods of neuroprotection. The events that occur after an episode of ischemia are many and hypothermia can affect different steps of this cascade. The mechanisms of action of TH are varied and the possible explanation for the benefits of this therapy is probably the multiple mechanisms of action blocking the cascade of ischemia on many levels. TH can affect many metabolic pathways, reactions of inflammation, apoptosis processes, and promote neuronal integrity. To know the mechanisms of action of TH will allow a better understanding about the indications for this therapy and the possibility of searching for other therapies when used in conjunction with hypothermia will provide a therapeutic synergistic effect. ${ }^{6}$

There is some evidence that hypothermia induces an antiinflamatory cytokine profile, inhibits the formation of nitric oxide during endotoxemia and neutrophil aggregation in the rat. Also hypothermia attenuates endotoxin induced over expression of nitric oxide in rat lungs. ${ }^{7}$

Along with free radicals production, after an ischemic insult, inflammatory mechanisms emerge and may last hours to several days. This is a normal physiological response; there are cytokines that have been shown to be protective and other cytokines that are deleterious such as TNF-alpha and IL-1Beta. ${ }^{8}$ Hypothermia decreases the production of inflammatory cytokines, leukotrienes, and inflammatory cells function such as macrophages. ${ }^{9}$ Moderate hypothermia delays the induction of proinflammatory cytokines in human peripheral blood mononuclear cells. ${ }^{10}$

A recent study compared the effects of mild and moderate hypothermia on endotoxemia in rats. Study showed that moderate hypothermia, in contrast to normothermia and deep hypothermia, produced anti-inflammatory effects, including reduction of leukocyte mobilization and inhibition of TNF- $\alpha$ production during cardiac surgery. Also both moderate and mild hypothermia improved mortality rates and attenuated endotoxin-induced changes, including attenuation of TNF- $\alpha$, IL-6, and NOx production. ${ }^{11}$

\section{Consequences of the hypothesis}

It is essential that before embarking on a human study, animal studies should be consideredas proof of principle study to support this hypothesis. If my hypothesis is true, it will improve obstetric outcome by lengthening pregnancy and thus reducing the incidence of preterm delivery (before 34 weeks gestation), improve neonatal outcome by reducing major morbidity and death, improve childhood cognitive and neurological outcome.

\section{Acknowledgments}

None.

\section{Conflicts of interest}

The authors declare there is no conflict of interests.

\section{References}

1. Romero R, Espinoza J, Gonçalves LF, et al. The role of inflammation and infection in preterm birth. Semin Reprod Med. 2007;25(1):21-39.

2. Romero R, Espinoza J, Gonçalves LF, et al. Inflammation in preterm and term labour and delivery. Semin Fetal Neonatal Med. 2006;11(5):317326.

3. van der Heyden JL, van Kuijk SM, van der Ham DP, et al. Subsequent Pregnancy after Preterm Prelabor Rupture of Membranes before 27 Weeks' Gestation. AJP Rep. 2013;3(2):113-118.

4. Abbott D, To M, Shennan A. Cervical cerclage: a review of current evidence. Aust N Z J Obstet Gynaecol. 2012;52(3):220-223.

5. Norman JE, Shennan A, Bennett P, et al. Trial protocol OPPTIMUM-does progesterone prophylaxis for the prevention of preterm labour improve outcome? BMC Pregnancy Childbirth. 2012;12:79.

6. González-Ibarra FP, Varon J, López-Meza EG. Therapeutic hypothermia: critical review of the molecular mechanisms of action. Front Neurol. 2011;2:4.

7. Scumpia PO, Sarcia PJ, Kelly KM, et al. Hypothermia induces antiinflammatory cytokines and inhibits nitric oxide and myeloperoxidasemediated damage in the hearts of endotoxemicrats. Chest. 2004;125(4):1483-1491.

8. Merrill JE, Benveniste EN. Cytokines in inflammatory brain lesions: helpful and harmful. Trends Neurosci. 1996;19(8):331-338.

9. Gu LJ, Xiong XX, Ito T, et al. Moderate Hypothermia Inhibits Brain Inflammation and Attenuates Stroke-Induced Immunodepression in Rats. CNS Neurosci Ther. 2013;20(1):67-75.

10. Kimura A, Sakurada S, Ohkuni H, et al. Moderate hypothermia delays proinflammatory cytokine production of human peripheral blood mononuclear cells. Crit Care Med. 2002;30(7):1499-1502.

11. Taniguchi T, Kanakura H, Takemoto Y, et al. Effects of hypothermia on mortality and inflammatory responses to endotoxin-induced shock in rats. Clin Diagn Lab Immunol. 2003;10(5):940-943. 